\title{
Thermodiffusion of monovalent organic salts
}

\section{in water}

\author{
André Luiz Sehnem, ${ }^{\dagger}$ Doreen Niether, ${ }^{\ddagger}$ Simone Wiegand, ${ }^{\ddagger}, \mathbb{\Phi}$ and Antônio Martins \\ Figueiredo Neto*,† \\ $\dagger$ Institut of Physics, University of São Paulo, Brazil \\ $\ddagger I C S$-3 Soft Condensed Matter, Forschungszentrum Jülich GmbH, D-52428 Jülich, \\ Germany \\ \Department für Chemie - Physikalische Chemie, Universität zu Köln, 50939 Cologne, \\ Germany \\ E-mail: afigueiredo@if.usp.br
}

\begin{abstract}
The ionic Soret effect induced by temperature gradients is investigated in organic electrolytes (tetramethylammonium and tetrabutylammonium hydroxides) dispersed in water using a holographic grating experiment. We report the influences of temperature and salt concentrations on the Soret, diffusion and thermal diffusion coefficients. Experimental results to the thermal diffusion coefficient are compared with a theoretical description for thermodiffusion of Brownian particles in liquids based in the thermal expansion of the liquid solution. It is observed that the obtained thermal diffusion coefficients for the organic electrolytes present a similar temperature dependence as the theoretical prediction. Comparing the experimental results for the organic and common inorganic salts it is proposed an additional physical mechanism as the cause to the different thermal diffusion coefficients in both types of salt. We propose that the temperature dependence of hydration free energy gives rise to a force term that also leads
\end{abstract}


to ion migration in a temperature gradient. We describe the thermal diffusion results as a competition between thermal expansion and hydration effects. The specific structure each type of ion cause in water molecules is considered in the heat of transport theory to describe thermal diffusion of electrolytes. A qualitative agreement is seen between our results and the classical heat of transport theory.

February 2, 2018

\section{INTRODUCTION}

In recent years it has been shown that Soret effect plays a significant role in the solute transport of aqueous solutions containing biological molecules and nanoparticles. ${ }^{1-3}$ Results are strongly influenced by the addition of salt and its specific nature (order-making or disrupting the water structure). ${ }^{4}$ The thermodiffusion effect due to the presence of temperature gradient in aqueous solutions of the organic salts tetramethylammonium and tetrabutylammonium hydroxides (TMAOH $-\mathrm{C}_{4} \mathrm{H}_{13} \mathrm{NO}$ and $\mathrm{TBAOH}-\mathrm{C}_{16} \mathrm{H}_{37} \mathrm{NO}$, respectively) is discussed in this work. These organic ions are extensively used as model systems to investigate how hydrophobic and electrostatic interactions influence hydration. ${ }^{5-7}$ Both organic electrolytes are used to disperse iron oxide nanoparticles to form stable ferrofluids ${ }^{8,9}$ but the hydration contribution of the salt to the Soret effect (either in oxide particles or aqueous solutions of the salts) is not clear. This contribution will be investigated by the experimental measurement of the Soret coefficient $S_{\mathrm{T}}$ (defined below) of the salts in aqueous solutions, and by comparing the results to the values previously obtained for alkali halide salts. ${ }^{10}$

The Soret effect is known to drive motion of solutes in mixtures and liquid dispersions in temperature gradients, creating nonhomogeneous distribution of the solute in the mixture. ${ }^{1,3,11,12}$ A phenomenological way to describe the effect in a binary mixture or liquid dispersion is to associate a thermophoretic flux $\mathbf{j}$ to the moving solute $\mathbf{j}=c_{i}\left(1-c_{i}\right) D_{\mathrm{T}} \delta T$. $D_{\mathrm{T}}$ is the thermal diffusion coefficient and $c_{i}$ the concentration of the solute in the tempera- 
ture gradient $\delta T$. The created concentration gradient $\delta c_{i}$ induces a counterflow due to Fick's law of diffusion given by $j=-D \delta c_{i}$, with the mass diffusion coefficient of the solute $D$. When both fluxes have the same magnitude and the total flux is null, the Soret coefficient is defined as

$$
S_{\mathrm{T}}=\frac{D_{\mathrm{T}}}{D}=-\frac{1}{c_{i}\left(1-c_{i}\right)} \frac{\delta c_{i}}{\delta T}
$$

and it is positive when the solute moves to the cold side and negative when it moves to the hot side.

The thermodiffusion behavior for monovalent ions and small molecules dispersed in water are of concern here, as these are physical representations for our systems. After the discovery of the thermodiffusion effect in salt solutions ${ }^{11}$, much research has been conducted to describe the phenomena in aqueous solutions of salts, ${ }^{13-17}$ molecules, ${ }^{18 ?-22}$ polymers ${ }^{23,24}$ and particles. ${ }^{25,26}$ So far there is no general theory to describe the phenomena since every dispersed solute presents its specific thermodiffusion behavior in any solvent. Some general trends are observed related to solute characteristics (size, molecular weight) and with experimental parameters (solute concentration and sample temperature).

The influences of temperature and ion concentration are the subjects here. At very low ion concentration of $\mathrm{NaCl}$ and $\mathrm{KCl}$ in water, Gaetaet al ${ }^{13}$ observed an unsual sharp minimum in the Soret coefficient, also observed by Colombani et al. ${ }^{27}$ for aqueous LiCl. These observations have been related to water structure changes in hydration shell and bulk water as the salt concentration changes. Also the temperature dependence $S_{\mathrm{T}}(T)$ in aqueous systems has been widely investigated for many solutes, showing a similar behavior: $2,15,19,21,22,25,28,29$ $S_{\mathrm{T}}(T)$ increases with temperature, for some systems showing $S_{\mathrm{T}}<0$ at low temperature and a sign inversion in the temperature range of experiments (usually $5{ }^{\circ} \mathrm{C}<T<60{ }^{\circ} \mathrm{C}$ ). In some systems, these changes in $S_{\mathrm{T}}$ induced by concentration and temperature were associated to the higher trend for water molecules to form hydrogen bond network at lower temperatures and concentrations. ${ }^{10,19}$ However there is no theoretical description which may be used to describe the most of the experimental results. 
A theoretical framework for thermal diffusion is the kinematical model of Brownian particles developed by H. Brenner and J. R. Bielenberg. ${ }^{30,31}$ The authors show that due to thermal expansion of a liquid solution when a temperature gradient is present, a volume element of the solution feels a thermophoretic force $\mathbf{F}=-\left(D_{\mathrm{th}} \beta / \mu\right) \nabla T$ and resulting in a velocity $\mathbf{U}$ given by $\mathbf{U}=-D_{\mathrm{th}} \beta \nabla T$. $D_{\text {th }}$ is the thermal diffusivity, $\beta$ the thermal expansion coefficient of the solution and $\mu$ the friction coefficient $\left(\mu=(6 \pi \eta a)^{-1}\right.$ for a spherical particle of radius a). The velocity $\mathbf{U}$ is against the temperature gradient for $\beta>0$, which is the case in most part of liquids, implying that solute migrates towards the cold side. A simple equation for the thermal diffusion coefficient holding for infinite dilution is given by $D_{\mathrm{T}}=\beta D_{\mathrm{th}}$, which we compare with experimental results.

We present experimental results to the Soret coefficient $S_{\mathrm{T}}$ and mass diffusion $D$ as function of ion concentration and temperature for aqueous solutions of TMAOH and TBAOH. The calculated values for $D_{\mathrm{T}}=D S_{\mathrm{T}}$ are presented as well. Both $S_{\mathrm{T}}$ and $D$ are obtained by the holographic grating experiment (Infra Red Thermal Diffusion Forced Rayleigh Scattering), ${ }^{32,33}$ from amplitude and build up time of the concentration grating respectively. A brief discussion is made about the concentration dependence as the results show no and low dependence for TMAOH and TBAOH. The calculated results for $D_{\mathrm{T}}(T)$ are compared with the theoretical result $D_{\mathrm{T}}(T)=\beta(T) D_{\text {th }}(T)$, and with $D_{\mathrm{T}}(T)$ values obtained from experiments of aqueous alkali halide solutions. ${ }^{10}$ It is observed that the agreement between our data and the single theoretical curve depends on the type of salt, as the results for the organic electrolytes are closer to the theoretical curve compared to the alkali halides. We will justify that the agreement is related to the different ways each ion organizes water molecules in the hydration shells. The presence of a temperature gradient originates a force described by the hydration free energy due to the temperature dependence of the ion/water interaction potential and entropy. In this way, our approach will be useful to describe thermal diffusion transport of salt solutions from the microscopic point of view. 


\section{EXPERIMENTAL SECTION}

The aqueous solutions of TMAOH and TBAOH were purchased from Sigma Aldrich at concentrations of $25 \mathrm{wt} \%$ and $1 \mathrm{M}$, respectively. A series of more diluted solutions was prepared by mixing the original solutions with Mili-Q water and stirring the solution for 10 minutes. The lowest concentrations investigated $(4 \mathrm{mM}$ for TMAOH and $2 \mathrm{mM}$ for TBAOH) are limited by the signal-to-noise ratio in IR-TDFRS measurement signal. The highest concentration was $0.2 \mathrm{M}$ and $0.1 \mathrm{M}$ for $\mathrm{TMAOH}$ and $\mathrm{TBAOH}$, which agrees with the concentration range used for dispersing iron oxide nanoparticles. ${ }^{8}$

Optical contrast factors $(\partial n / \partial c)_{p, T}$ and $(\partial n / \partial T)_{p, c}$ were determined by measuring the refractive index in the temperature range $20{ }^{\circ} \mathrm{C}<T<60{ }^{\circ} \mathrm{C}$. A refractometer (ATAGO 5000i), with a light source from a sodium lamp with $\lambda=589 \mathrm{~nm}$, and temperature control $\triangle T=0.01 \mathrm{~K}$ is used to measure the temperature and concentration dependence of the refractive index. The temperature dependence of $(\partial n / \partial T)_{p, c}$ maintained a similar behavior as that for pure water. Values obtained for $(\partial n / \partial c)_{p, T}$ are independent of temperature $(0.19$ $\pm 0.01 / \mathrm{M}$ and $0.12 \pm 0.01 / \mathrm{M}$ for TMAOH and TBAOH, respectively).

A cell with quartz glass (Hellma) with $0.2 \mathrm{~mm}$ optical path length is used as sample cell in the IR-TDFRS experiments. All solutions were filtered with a nylon filter with a diameter of $5 \mu \mathrm{m}$ to avoid dust particles. The sample holder is inserted inside a copper cell that allows water circulation for temperature control $(\triangle T=0.05 \mathrm{~K})$. A small window in the copper cell allows the laser passage through the sample. The experiments are repeated at least twice for each temperature to validate the reproducibility of the results.

\section{IR-TDFRS Setup}

The details about the Thermal Diffusion Forced Rayleigh Scattering technique can be found elsewhere $^{33-35}$. An important detail is that in this work the laser beam used to induce the periodic temperature profile has a wavelength $\lambda_{w}=980 \mathrm{~nm}$ and is absorbed by the water 
molecules to generate the temperature and concentration gradients. A He-Ne laser with wavelength $\lambda_{r}=632.8 \mathrm{~nm}$ is used as read-out beam. ${ }^{19,32}$ This technique can be used for different solutes in water, as long as the solute shows a refractive index contrast related to that of water. ${ }^{10,36}$

A brief description of the technique is the following: the infrared laser is split in two beams before passing the sample. They intersect the same spot in the sample, generating a sinusoidal periodic intensity distribution. This periodic intensity grating is controlled by the phase difference between the two beams generated by a piezoelectric mirror in one of the beams. As a consequence of light absorption a periodic temperature profile is generated in the sample and due to the Soret effect the solute particles move either to the maximum or minimum of temperature. Both the temperature and the concentration grating diffract the read-out beam. The build-up of the temperature grating is much faster compared to the concentration grating, as the thermal diffusivity $D_{\text {th }}$ is several orders of magnitude faster compared to the mass diffusion $D$. The amplitude of the total heterodyne scattered signal $\zeta_{\text {het }}(t)$ is normalized by the thermal grating signal and its final amplitude is related with the Soret coefficient. It is given by

$$
\zeta_{\text {het }}(t)=1-\exp \left(-\frac{t}{t_{\text {th }}}\right)-\frac{A}{\tau-\tau_{\text {th }}}\left[\tau\left[1-\exp \left(-\frac{t}{\tau}\right)\right]-\tau_{\text {th }}\left[1-\exp \left(-\frac{t}{\tau_{\text {th }}}\right)\right]\right]
$$

where $\tau_{\text {th }}$ is the heat diffusion time defined as $1 /\left(q^{2} D_{\mathrm{th}}\right), \tau$ is the Soret effect time defined as $1 /\left(q^{2} D\right), q$ is the scattering factor that can be obtained from the measured fringe spacing $d, d=2 \pi / q$. The amplitude $A$ is is given by

$$
A=\frac{(\partial n / \partial c)_{p, T}}{(\partial n / \partial T)_{p, c}} S_{\mathrm{T}} c(1-c)
$$

The best fitting of the equation 2 to the experimental curves $\zeta_{\text {het }}(t)$ results in values for $D_{\mathrm{th}}$, $D$, and $S_{\mathrm{T}}$. The thermal diffusion coefficient is calculated by $D_{\mathrm{T}}=D S_{\mathrm{T}}$. 


\section{EXPERIMENTAL RESULTS}

The discussion about the results obtained for $S_{\mathrm{T}}, D$, and $D_{\mathrm{T}}$ will be made in two sections. In the first one, the results for the concentration dependence at the highest and lowest temperatures are discussed. We compare with literature results and discuss to which extent ion concentration induce changes the hydration shell and can influence the concentration dependence of the Soret coefficient $S_{\mathrm{T}}(c)$. In the second section the data will be presented as function of temperature, which is a discussion topic per se in the thermodiffusion field and gives additional information about the strength of the hydrogen bonds in the system. A comparison between results and theoretical prediction by Brenner and Bielenberg theory ${ }^{31}$ is presented and will enlighten the physical mechanisms driving Soret effect in aqueous electrolytes.

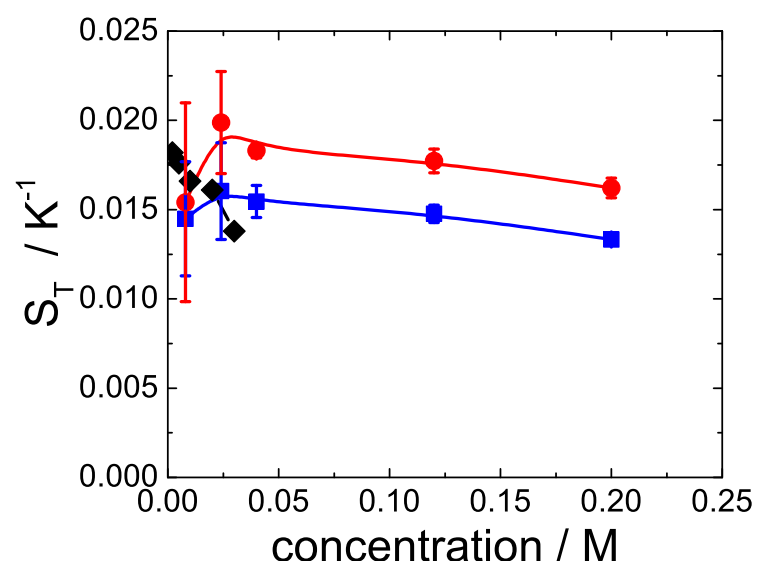

(a)

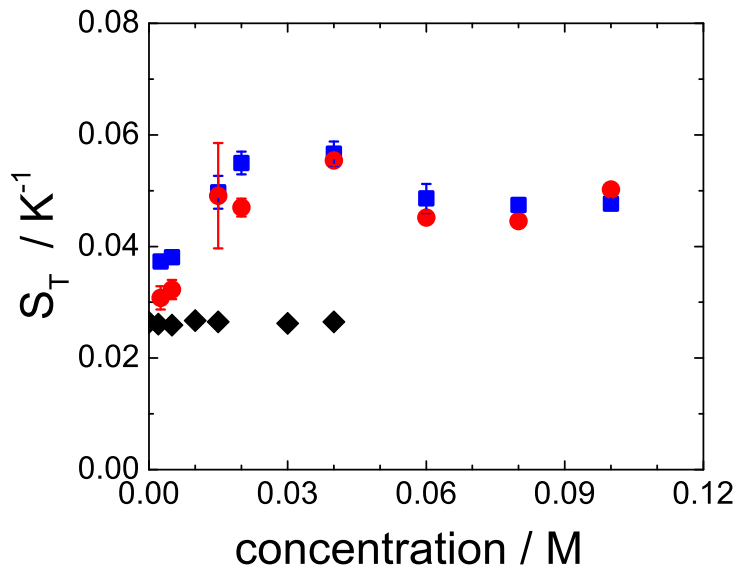

(b)

Figure 1: Soret Coefficient as a function of the electrolyte concentration for aqueous solutions of (a) TMAOH and (b) TBAOH. Blue squares represent $T=20^{\circ} \mathrm{C}$ and red circles $T=60^{\circ} \mathrm{C}$. Diamond symbols represent data published by Leaist and $\mathrm{HaO}^{14}$ for the same systems at $T=25^{\circ} \mathrm{C}$. Lines in (a) are guides to the eyes. 


\section{(i) Concentration dependence.}

Figures $1 \mathrm{a}$ and $1 \mathrm{~b}$ show the ion concentration dependence of the Soret coefficient $\left(S_{\mathrm{T}}(c)\right)$ for both TMAOH and TBAOH aqueous solutions, respectively. The results for the lowest $\left(20{ }^{\circ} \mathrm{C}\right)$ and highest $\left(60{ }^{\circ} \mathrm{C}\right)$ temperatures investigated are shown. In the case of TBAOH it shows a shallow maximum value of $S_{\mathrm{T}}$ at $c^{i} \approx 0.03 \mathrm{mM}$ and becomes constant at high concentrations while in the case of TMAOH a maximum is not evident due to the large error bars at low concentrations (low IR-TDFRS signal). For comparison we insert experimental results obtained by Leaist and Hao ${ }^{14}$ using Agar-Turner type conductivity cell. While for TMAOH both data sets agree within the large error bars at low concentration, our values for TBAOH are up to two times higher. Furthermore they observed a decreasing of $S_{\mathrm{T}}$ with increasing concentration of TMAOH and no concentration dependence for TBAOH.

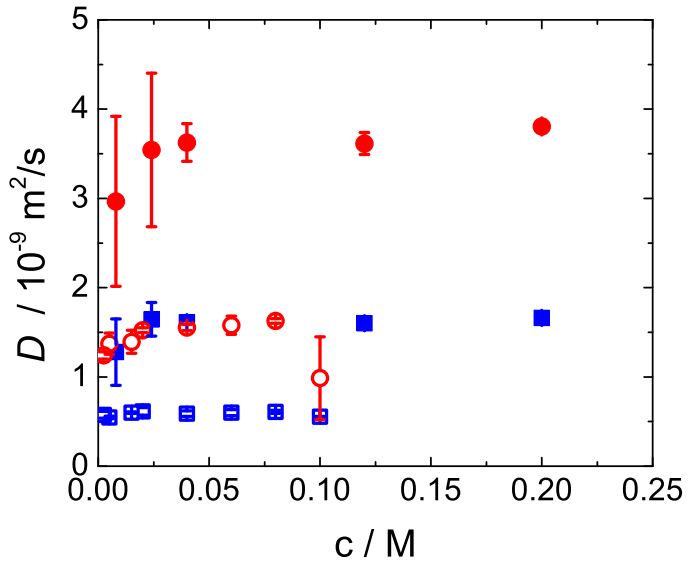

(a)

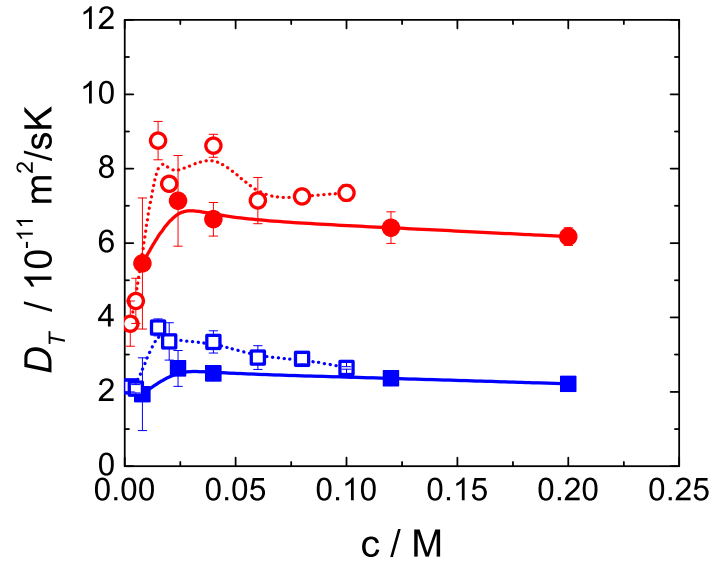

(b)

Figure 2: (a) Mass diffusion coefficient $D$ and (b) thermal diffusion coefficient $D_{\mathrm{T}}$ for TMAOH (solid symbols) and TBAOH (open symbols) at $T=20^{\circ} \mathrm{C}$ (blue) and $T=60^{\circ} \mathrm{C}$ (red); Black squares are results obtained by Leaist and Hao. ${ }^{14}$ The lines are guides to the eyes.

The results for $D(c)$ and $D_{\mathrm{T}}(c)$ of TMAOH and TBAOH solutions are shown in Figure $2 \mathrm{a}$ and $2 \mathrm{~b}$, respectively. The nearly constant values for $D$ indicate that the solutions are in the dilute regime, and ion-ion interactions do not occur in the investigated concentration range. It is expected that when the hydrophobic ions interact the diffusion values decrease 


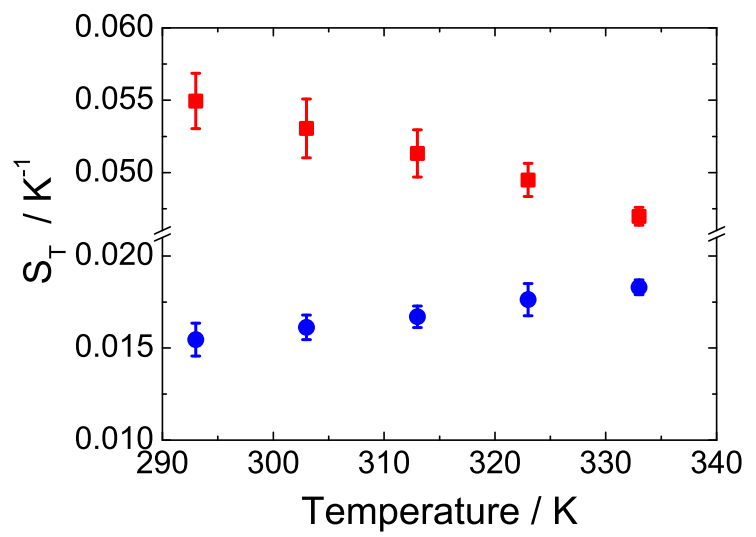

Figure 3: Temperature dependence of the Soret coefficient for TMAOH (circles) and TBAOH (squares) at low ionic concentration $c \sim 0.024 \mathrm{~mol} / \mathrm{L}$ and $0.02 \mathrm{~mol} / \mathrm{L}$, respectively.

with increasing concentration. ${ }^{5}$ The values obtained for $D$ at low temperature are around $30 \%$ lower than that obtained by Leaist and $\mathrm{Hao}^{14}$ for $\mathrm{TMAOH}$ and $\mathrm{TBAOH}$ at the same temperature. The thermal diffusion coefficient in Figure $2 \mathrm{~b}$ shows a similar behavior as $S_{\mathrm{T}}$ in Figure 1b. Two major points must be emphasized: (i) compared to other systems the obtained thermal diffusion coefficient $D_{\mathrm{T}}$ are higher as it is typically obtained $D_{\mathrm{T}}<$ $1 \times 10^{-11} \mathrm{~m}^{2} / \mathrm{sK} ;{ }^{19,37}$ (ii) the $D$ and $D_{\mathrm{T}}$ increase with temperature, as expected for $D$ due to the lower viscosity at higher temperatures. The description for temperature dependence of $D_{\mathrm{T}}$ will be discussed below.

\section{(ii) Temperature dependence}

Figure 3 shows $S_{\mathrm{T}}(T)$ for TMAOH and TBAOH at ion concentrations of $0.024 \mathrm{~mol} / \mathrm{L}$ and $0.02 \mathrm{~mol} / \mathrm{L}$ respectively. The temperature dependence for $S_{\mathrm{T}}$ observed in the case of TMAOH is similar to the typical behavior discussed in the introduction, while $S_{\mathrm{T}}$ decreases with increasing temperature for TBAOH. This inverse behavior for $S_{\mathrm{T}}$ is not common for aqueous systems, but it has been observed in some systems: ethanol/water, ${ }^{38}$ crown ether/ water ${ }^{39}$ and acetylsalicylic acid ${ }^{40}$ and will be discussed with the analysis of $D(T)$ and $D_{\mathrm{T}}(T)$ data. Although for many aqueous solution $S_{\mathrm{T}}$ changes from negative to positive with increasing temperature, ${ }^{2}$ this is not the case for TMAOH and TBAOH as both electrolytes are thermo- 
phobic in the entire temperature range. Even the extrapolation of the results to very high and very low temperatures does not suggest a sign inversion in the range $0{ }^{\circ} \mathrm{C}<T<100^{\circ} \mathrm{C}$.

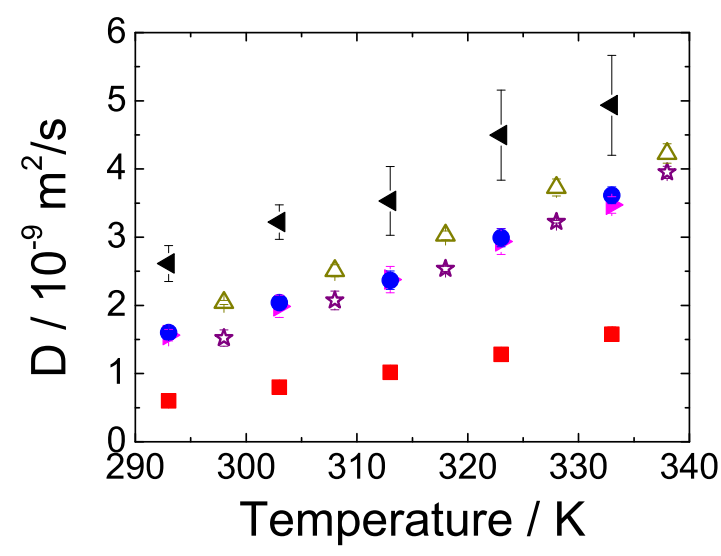

(a)

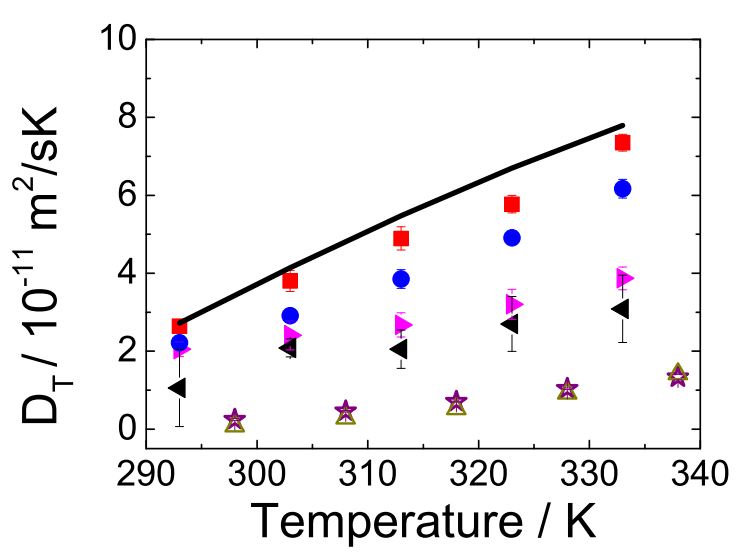

(b)

Figure 4: Temperature dependence of (a) mass diffusion coefficient $D$ and (b) thermal diffusion coefficient $D_{\mathrm{T}}$ for TBAOH (red squares) and TMAOH (blue circles) for ion concentrations $c=0.02 \mathrm{~mol} / L$ and $c=0.024 \mathrm{~mol} / L$, respectively; for $\mathrm{NaOH}$ (pink triangles) and $\mathrm{HNO}_{3}$ (black triangles) at concentration $c \sim 0.5 \mathrm{~mol} / L$ and the values obtained for $\mathrm{NaCl}$ (violet stars) and $\mathrm{KCl}$ (olive green triangles) from Römer et al ${ }^{10}$ at $c \sim 0.5 \mathrm{~mol} / \mathrm{L}$. Also shown in (b) is the theoretical calculation for $D_{\mathrm{T}}=\beta D_{\text {th }}$ (solid line).

Figure 4a shows that $D(T)$ values increase with temperature, as expected due to decreasing water viscosity. The noticeable difference between $D(T)$ of TMAOH and TBAOH is caused by the different cations sizes. An interesting information is the similar behavior $D(T)$ for TMAOH with monoatomic electrolytes, indicating that the small alkali-halide ions have water molecules attached as they diffuse implying in similar $D(T)$ values as the bigger ions of TMAOH. Figure $4 \mathrm{~b}$ presents $D_{\mathrm{T}}(T)$ results for both organic electrolytes and also for some common electrolytes. These results will be discussed in the next section and together with $D(T)$ results it will be possible to explain the positive and negative slopes observed for $S_{\mathrm{T}}(T)$.

The $D_{\mathrm{T}}(T)$ data for $\mathrm{NaOH}$ and $\mathrm{HNO}_{3}$ that stand in between the data for $\mathrm{NaCl} / \mathrm{KCl}$ and the organic electrolytes was also measured by us. It was mainly measured to establish a comparison of the data from at least six different eletrolytes, and will be useful for the following discussion. 


\section{DISCUSSION}

The concentration dependence of $S_{\mathrm{T}}$ reflects the $D_{\mathrm{T}}$ behavior as $D$ is constant in the concentration range of our experiments. In the next paragraph we discuss that it is not possible to get a full physical picture of the hydration contribution for the thermal diffusion effect as changes with concentration are much smoother than the changes observed in previous works of Gaeta et $a l^{13}$ and Colombani et $a l^{27}$ for alkali-halide ions. However, the temperature dependence of $D_{\mathrm{T}}(T)$ for TBAOH/TMAOH and their resemblance with Brenner's theoretical model for $D_{\mathrm{T}}(T)$ suggests the thermal expansion force being a major contribution to the Soret effect of such electrolytes. In contrast, experimental results of $D_{\mathrm{T}}(T)$ for $\mathrm{NaCl} / \mathrm{KCl}$ are roughly $80 \%$ below the theoretical values for high temperatures and more than $90 \%$ for low temperatures. These results will be described below based on the different hydration layers that organic and alkali-halide ions form. As Figure 4a shows, the temperature dependence for $D$ is similar for most of the investigated systems and does not have a clear correlation with results for $D_{\mathrm{T}}$. This fact suggests that results for $D_{\mathrm{T}}(T)$ may be described based on different approaches rather than that based on the gradient of the diffusion $D .{ }^{41,42}$

To discuss the influence of the orientation of the water molecules in the hydration layer of $\mathrm{TMA}^{+} / \mathrm{TBA}^{+}$on $S_{\mathrm{T}}(c)$ we follow the argumentation by Gaeta et al. ${ }^{13}$ to interpret their results in aqueous solutions of $\mathrm{NaCl}$ and $\mathrm{KCl}$. In the case of organic ions their hydrophobic nature induce the nearest water molecules to organize in a tangential orientation around them, ${ }^{5,7}$ while electrostatic interaction is clearly predominant between monoatomic ions/water molecules. ${ }^{44}$ This is illustrated in Figure 5, showing that an imaginary orientation axis of the $\mathrm{O}-\mathrm{H}$ water bonds (black lines in the figure) usually lies in a perpendicular direction related to the centre of the hydrophobic ions. As a response to different hydration one could expect that concentration and temperature effects reflected in $S_{\mathrm{T}}$ behavior of the organic electrolytes would differ for alkali and organic ions. However, the maximum value for $S_{\mathrm{T}}(c)$ is only very shallow in the case of TBAOH and does not appear for TMAOH, in contrast to the sharp minima observed by Gaeta et $a l^{13}$ for $\mathrm{NaCl}$ and $\mathrm{KCl}$ in similar 


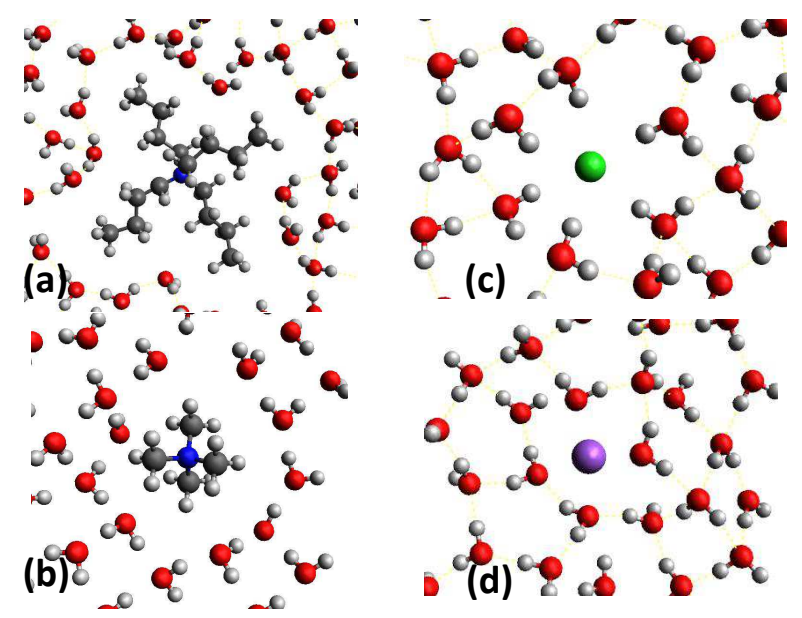

Figure 5: Illustrative images to show the water molecules structuring around some of the ions investigated. The images depict the most common results found in literature: ${ }^{43,44}$ the gas-like interface of $\mathrm{TBA}^{+}$and $\mathrm{TMA}^{+}$ions ( $\mathrm{a}$ and $\mathrm{b}$ ) with the $\mathrm{O}-\mathrm{H}$ water bonds lying tangentially to the ions interface showing low electrostatic interaction (black lines are parallel to the $\mathrm{O}-\mathrm{H}$ water bonds). Illustrations (c) and (d) show water around monoatomic ions. In this case molecules clearly align radially with hydrogens toward negative (c) and oxygen toward positive (d) ion. The influence of these water organization on the thermal diffusion coefficient $D_{\mathrm{T}}$ is discussed in the text.

concentration range. In the more recent work $^{10}$ the very low concentration range was not accessible, but for higher concentrated solutions of $\mathrm{NaCl}$ and $\mathrm{KCl}$ a qualitative agreement between experiments and molecular dynamics simulations was found. The gain a microscopic understanding of the ion hydration on the Soret coefficient more systematic studies are required.

Now we explain the different slopes in $S_{\mathrm{T}}(T)$ curves in Figure 3 . Since $S_{\mathrm{T}}$ is the ratio of $D_{\mathrm{T}} / D$ and $D_{\mathrm{T}}(T)$ curves for both organic salts are similar while $D(T)$ results are rather different, it suggests that a first comparison with a thermodiffusion theory for $D_{\mathrm{T}}$ must be done with a theoretical description that does not consider specific properties of the solute as its size. This is the case of the hydrodynamic theory for Brownian particles developed by Bielenberg and Brenner, ${ }^{30,31}$ briefly described in the introduction. The theoretical expression for thermal diffusion coefficient $D_{\mathrm{T}}$ is given by $D_{\mathrm{T}}=D_{\mathrm{th}} \beta$. As we are working in the low concentration regime of electrolytes, we assume that $D_{\text {th }}$ and $\beta$ are approximately equal to the water values (this approximation was verified for $D_{\text {th }}$ from values obtained by the best 
fitting of equation 2 to the IR-TDFRS results). The result from the theoretical calculation of $D_{\mathrm{T}}$ in the investigated temperature range is shown as solid line of Figure $4 \mathrm{~b}$. The similarity between experimental results for $D_{\mathrm{T}}(T)$ in both organic electrolytes implies that the crucial factor to the $S_{\mathrm{T}}(T)$ behavior (positive or negative $T$-slope) are values in the curve $D(T)$. One can observe in Figure 4a that the values and $T$-slope for TBAOH are smaller compared to TMAOH. Then the big size of $\mathrm{TBA}^{+}$imply in a negative $S_{\mathrm{T}}(T)$-slope for this ion and positive to the smaller ion of $\mathrm{TMA}^{+}$.

In recent works ${ }^{40,45}$ it was argued that the slopes of the temperature dependence for $S_{\mathrm{T}}(T)$ are related to the hydrophilicity of the solutes. For the uncharged systems they found that the change of the Soret coefficient between a higher (e.g. $\left.50^{\circ} \mathrm{C}\right)$ and lower (e.g. $20^{\circ} \mathrm{C}$ ) temperature is larger for more hydrophilic systems with a large negative value of $\log P$. This is a measure for hydrophilicity/hydrophobicity of a solute used to model the transport of a compound in the environment or to screen for potential pharmaceutical compounds.?

Comparing the experimental $D_{\mathrm{T}}(T)$ results with the theoretical value by Brenner in Figure $4 \mathrm{~b}$ the experimental values are below the theoretical line. Note that the $\mathrm{NaCl}$ and $\mathrm{KCl}$ results by Römer et al. ${ }^{10}$ have been measured at a concentration of $0.5 \mathrm{~mol} / \mathrm{L}$. Extrapolating the values to the lower concentrations measured for the organic electrolytes would lead to even lower $D_{\mathrm{T}}(T)$ values increasing the difference between the experimental values and the theoretical line. The organic electrolytes show the best agreement with the theoretical predictions. It means that the thermal expansion contribution to the thermal diffusion coefficient is weakened or counterbalanced by other physical effects in alkali halide ions.

The main difference between tetraalkylammonium and alkali halide salts is the hydrophobic nature of the organic ions. It is known that the monoatomic ions induce water ordering by dipole reorientation and influence the hydrogen bonding network in the first and second hydration shells, compared to that in bulk water. ${ }^{13,46}$ The halide anions even form strong hydrogen bonds with water molecules, with coordination number up to $6{ }^{47}$ In the case of poliatomic organic ions the interface ion/water molecules forms a gas-like interface for $\mathrm{TBA}^{+}$ 
ions (decreasing water-water hydrogen bonding around the ion) ${ }^{48}$ For $\mathrm{TMA}^{+}$there is a literature controversy, whether a gas-like interface is ${ }^{48,49}$ or is not formed. ${ }^{50}$ However molecular dynamic simulations of the organic ions in water indicate that water organization around both ions is very similar to that shown in Figure $5 .^{7,51}$ Based on this qualitative discussion, in next section we develop an argument to relate the thermal diffusion coefficient $D_{\mathrm{T}}$ with a general Gibbs free energy for ionic aqueous solutions.

\section{The Gibbs free energy in temperature gradient}

In the previous paragraphs we introduced the hypothesis that $D_{\mathrm{T}}$ may also be related to the hydration layer of the ions, which is expected to be different for the organic salts compared to the alkali halides. In order to develop a physical framework for the hydration layer contribution to the thermal diffusion we are adapting a concept used to describe the electric double layer contribution to the Soret effect by Dhont. ${ }^{52,53}$ Lets consider the cartoon displayed in Figure 6 . We want to displace a hydrated ion in a temperature gradient from a temperature $T$ by a distance $d z$ to a temperature $T+\delta T$. As a first step we remove the hydration layer, which requires the work $-W^{\text {hyd }}(T)$. Now we raise the temperature to $T+\delta T$, which does not cost any work. Finally we have to rebuild the hydration layer at a temperature $T+\delta T$, which cost the work $-W^{\text {hyd }}(T+\delta T)$.

In this picture, the work to remove and rebuild the hydration layer is derived from the Gibbs free energy due to hydration of ions by water molecules $\triangle G^{\text {hyd }}$. It is defined as $\triangle G^{h y d}=\triangle H^{h y d}-T \triangle S^{h y d}$ where $\triangle H^{\text {hyd }}$ is the enthalpy and $\triangle S^{h y d}$ the entropy of the hydration layer. Considering the dependence of temperature in one spacial coordinate $z$, we can express the free energy force $F_{f e}=-d\left(\triangle G^{h y d}\right) / d z-\left(d\left(\triangle G^{h y d}\right) / d T\right) d T / d z=-\left(\partial_{T}\left(\triangle G^{h y d}\right)\right) \nabla T$. The enthalpy is given by $H=U+p V$, as function of inner energy $U$, pressure $p$ and volume element $V$. As this force act upon ions, a displacement velocity will contribute to move them in the temperature gradient.

The situation in the electrolyte solution is rather complicated due to long-range electro- 


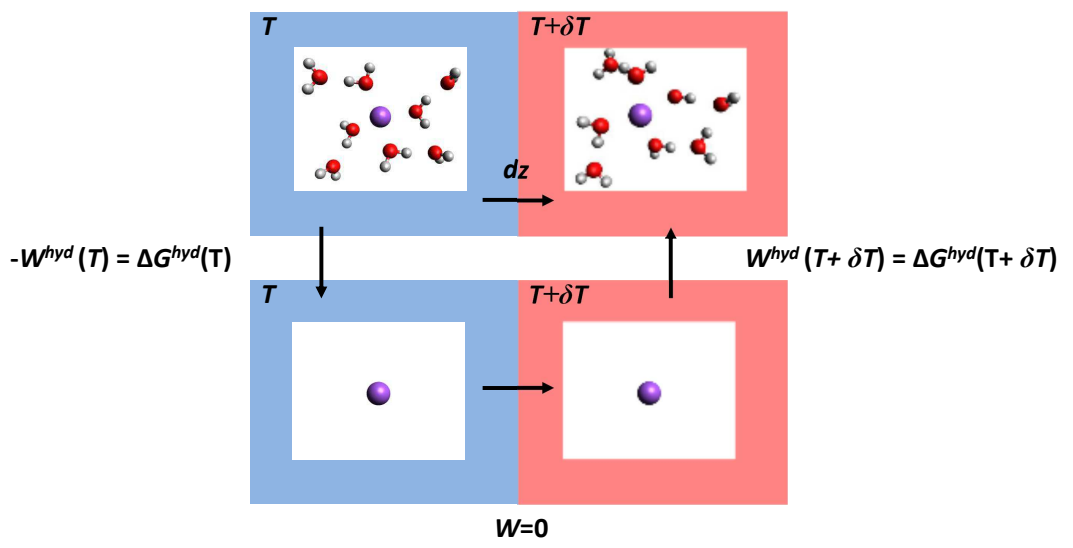

Figure 6: Sketch describing ion displacement by an amount $d z$ in a smooth temperature gradient in three steps. Firstly we remove the hydration layer around an ion performing a work $-W^{h y d}(T)=\triangle G^{h y d}(T)$. Then the ion is displaced by $d z$ to a position with temperature $T+\delta T$. Finally, re-building the hydration layer at $T+\delta T$ requires a work $W^{\text {hyd }}(T+\delta T)=$ $\triangle G^{\text {hyd }}(T+\delta T)$. 
static interactions and local variation of the dielectric constant due to re-arrangement of the water molecules around the ion.? ? We use here a simple electrostatic approach assuming the same dielectric constant as in bulk water. In the so-called Born model ${ }^{\text {? }}$ the energy of the electrostatic field around the ion given by $U_{\mathrm{el}}=e^{2} / 2 \varepsilon r$, where $e$ is the elementary charge, $\varepsilon$ the dielectric permittivity and $r$ the ionic radius. By dilution of a monovalent salt in water one obtains

$$
\Delta G^{\mathrm{hyd}}=\frac{e^{2}}{2}\left(\frac{1}{\varepsilon}-1\right)\left(\frac{1}{r_{+}}+\frac{1}{r_{-}}\right),
$$

leading to thermophoretic hydration layer force due to electrostatic interaction

$$
F_{\mathrm{fe}}^{\mathrm{el}}=\frac{e^{2}}{2}\left(\frac{1}{\varepsilon^{2}} \frac{d \varepsilon}{d T}\right)\left(\frac{1}{r_{+}}+\frac{1}{r_{-}}\right) \nabla T
$$

This result shows that when the liquid has $d \varepsilon / d T<0$, like water, the ion is pushed from the cold to the hot side by this force and that the force is more intense for a smaller ionic radius. This bring us to the conclusion that the big ions $\mathrm{TMA}^{+} / \mathrm{TBA}^{+}$are less affected than $\mathrm{NaCl} / \mathrm{KCl}$ by the force derived from the hydration layer.

It is known that hydration enthalpy and entropy have many contributions ${ }^{54}$ as the electrostatic, van der Waals and hydrophobic interactions between water and solutes (enthalpy) and translational, rotational and vibrational entropies. These properties are investigated by dynamic simulations for small molecules ${ }^{54}$ and ions, ${ }^{55}$ even as function of temperature. ${ }^{43,51,56}$ To compute the temperature dependence from all the free energy contributions in order to have a precise quantification to the force from the free energy of hydration layer $F_{\mathrm{fe}}$ would certainly be an interesting challenge, but it is beyond the scope of this work.

\section{Combining effects of thermal expansion and free energy}

The similarity between Brenner's theory and the experimental results for the organic electrolytes presented in Figure 4b suggests a positive contribution from thermal expansion to the ionic Soret effect. As stated in the previous paragraph we do have an additional con- 
tribution by the hydration layer moving the ions to the warm side and therefore leading to a negative contribution of $D_{\mathrm{T}}$, which becomes stronger for the small ions compared to the larger organic ions. Therefore we propose to expand Brenner's model ${ }^{30,31}$ to write the total thermal force

$$
F=F_{T}+F_{\mathrm{fe}} \propto-\left(\frac{\beta D_{\mathrm{th}}+D_{T}^{f e}}{\mu}\right) \nabla T
$$

leading to the final expression for the thermophoretic mobility $D_{T}=\beta D_{\mathrm{th}}+D_{\mathrm{T}}^{\mathrm{fe}}$ whereas a expression of $D_{\mathrm{T}}^{\mathrm{fe}}$ requires a better understanding of ionic solutions as function of temperature effects.

\section{Qualitative comparison with the heat of transport}

One of the main theories describing the Soret effect in electrolytes is related to the ionic heat of transport $Q_{i}^{*}$. This theory is based on the specific entropy change each ion induces in the surrounding solvent molecules moving through the temperature gradient. ${ }^{3,57}$ The Soret coefficient of an electrolyte solution is related to $Q_{i}^{*}$ by the relation

$$
Q_{i}^{*}=2 S_{\mathrm{T}} R T^{2}\left(1+\left(\frac{\partial \ln \gamma}{\partial \ln m}\right)\right)
$$

where $m$ is the molality, $\gamma$ the mean activity coefficient at the molality scale. In the case of monovalent electrolytes, $Q_{i}^{*}=Q_{+}^{*}+Q_{-}^{*}$.

The main tests for this theory are performed on comparing the experimental thermophoretic migration of micro and nanoparticles. The difference in the heats of transport of anions and cations $\left(Q_{-}^{*}-Q_{+}^{*}\right)$ gives rise to a macroscopic thermoelectric field that drives the particles of known surface charge either to cold or hot side. ${ }^{4,21,28,58-60}$ In this way the thermodiffusive effects of charged particles are related to the ionic heat of transport, although the microscopic effects that drive ions to move to cold or hot side is an enduring question.

In order to do some comparison between our picture of thermal diffusion and the heat of 
transport theory, we call attention to a resemblance between the measured $D_{\mathrm{T}}$ values for the systems at room temperature with the the heat of transport $Q^{*}$ of the ionic solutions in the literature. ${ }^{14,57,59,61}$ We expect the following order TBAOH $>\mathrm{TMAOH}>\mathrm{NaOH}>\mathrm{HNO}_{3}>$ $\mathrm{NaCl}>\mathrm{KCl}$ for $Q^{*}$, which coincide with the order of $D_{\mathrm{T}}$ shown in Figure $4 \mathrm{~b}$. Summarizing we state that Agar's theory of heat of transport ${ }^{57}$ as well as Brenner's theory ${ }^{30}$ based on the thermal expansion of the solution show qualitative agreement with our experimental results.

\section{CONCLUSION}

In this work the temperature dependence of the thermal diffusion coefficient $D_{\mathrm{T}}$ of two aqueous electrolytes TBAOH and TMAOH was experimentally investigated. We described that these electrolytes show higher thermal diffusion coefficients $D_{\mathrm{T}}$ compared to alkali-halide salts. Based on the Born approximation it is suggested that the temperature dependence of the hydration free energy for organic electrolytes and alkali-halide salts is stronger for the last systems. As this force caused by the formation of the hydration layer pushes the ions to the hot side and lowers the $D_{\mathrm{T}}(T)$-values predicted by Brenner's model based on thermal expansion of the solution. The temperature dependence of the Soret coefficient $S_{\mathrm{T}}(T)$ (positive slope for TMAOH and negative slope for TBAOH) is a consequence of the temperature dependence of the diffusion coefficient $D(T)$ due to different sizes of $\mathrm{TMA}^{+}$ and $\mathrm{TBA}^{+}$and similar dependence of $D_{\mathrm{T}}(T)$. We concluded that the physical picture based in the solvent thermal expansion and hydration free energy describes the results to the transport coefficient $D_{\mathrm{T}}$, in a qualitative agreement with the ionic heat of transport. This outcome agrees also with the intuitive physical pictures that the alkali-halide salts show stronger interactions with the hydration layer by forming hydrogen bonds (c.f. 5), so that it is expected that this systems show larger deviations from the ideal behavior. 


\section{Acknowledgement}

The authors are thankful with Dr. Hartmut Kriegs for his help with the experiments and with Dr. Jan K. G. Dhont for fruitful discussions. The authors acknowledge the financial support from CAPES-Br (Coordenação de Aperfeiçoamento de Pessoal de Nivel Superior - n 88881.133118/2016-01) and INCT-FCx (Instituto Nacional de Ciência e Tecnologia de Fluidos Complexos) (CNPq - n 465259/2014-6 and FAPESP - n²014/50983-3). Part of the experimental data presented were obtained with funding from the European Union's Horizon 2020 research and innovation programme under grant agreement No 731019 (EUSMI) which is gratefully acknowledged. DN acknowledges support by the International Helmholtz Research School of Biophysics and Soft Matter (IHRS BioSoft).

\section{References}

(1) Wiegand, S. Thermal diffusion in liquid mixtures and polymer solutions. J. Phys.: Condens. Matter 2004, 16, R357.

(2) Iacopini, S.; Rusconi, R.; Piazza, R. The "macromolecular tourist": universal temperature dependence of thermal diffusion in aqueous colloidal suspensions. Eur Phys J E Soft Matter 2006, 19, 59-67.

(3) Wurger, A. Thermal non-equilibrium transport in colloids. Rep. Prog. Phys. 2010, 73, 126601.

(4) Eslahian, K. A.; Maskos, M. Hofmeister effect in thermal field-flow fractionation of colloidal aqueous dispersions. Colloids and Surfaces A: Physicochemical and Engineering Aspects 2012, $\underline{413}, 65-70$.

(5) Bhowmik, D.; Malikova, N.; Mériguet, G.; Bernard, O.; Teixeira, J.; Turq, P. Aqueous solutions of Tetraalkylammonium halides: ion hydration, dynamics and ion-ion interactions in light of steric effects. Phys Chem Chem Phys 2014, 16, 13447-13457. 
(6) Marcus, Y. Tetraalkylammonium Ions in Aqueous and Non-aqueous Solutions. J. Solution Chem. 2008, 37, 1071-1098.

(7) Dong, D.; Hooper, J. B.; Bedrov, D. Structural and Dynamical Properties of Tetraalkylammonium Bromide Aqueous Solutions: A Molecular Dynamics Simulation Study Using a Polarizable Force Field. J. Phys. Chem. B 2017, 121, 4853 - 4863.

(8) Filomeno, C. L.; Kouyatè, M.; Peyre, V.; Demouchy, G.; Campos, A. F. C.; Perzynsky, R.; Tourinho, F. A.; Dubois, E. Tuning the Solid/Liquid Interface in Ionic Liquid Colloidal Dispersions: Influence on Their Structure and Thermodiffusive Properties.

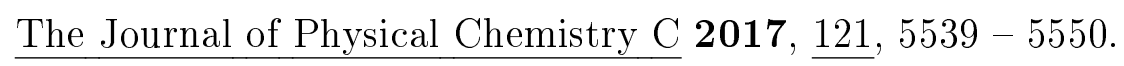

(9) Lucas, I. T.; Durand-Vidal, S.; Dubois, E.; Chevalet, J.; Turq, P. Surface Charge Density of Maghemite Nanoparticles: Role of Electrostatics in the Proton Exchange. J. Phys. Chem. C 2007, 111, 18568 - 18576.

(10) Römer, F.; Wang, Z.; Wiegand, S.; Bresme, F. Alkali Halide Solutions under Thermal Gradients: Soret Coefficients and Heat Transfer Mechanisms. The Journal of Physical Chemistry B 2013, 117, 8209-8222.

(11) Soret, C. Sur L'État D'Équilibre que Prend, du Point de Vue de sa Concentration, une Dissolution Saline Primitivement Homogéne, Dont Deux Parties Sont Portées à des Températures Différentes. Arch. Sci. Phys. Nat. Geneve 1879, 3, 48.

(12) Bringuier, E.; Bourdon, A. Colloid transport in nonuniform temperature. Phys. Rev. E 2003, 67,011404 .

(13) Gaeta, F. S.; Perna, G.; Scala, G.; Bellucci, F. Nonisothermal Matter Transport in Sodium Chloride and Potassium Chloride Aqueous Solutions. 1. Homogeneous Systems (Thermal Diffusion). The Jornal of Physical Chemistry 1982, 66, 2967-2974. 
(14) Leaist, D. G.; Hao, L. Heats of transport of aqueous tetraalkylammonium hydroxides and the electrophoretic effect. J. Chem. Soc., Faraday Trans. 1994, 90, 1223-1225.

(15) Longsworth, L. The Temperature Dependence of the Soret Coefficient of Aqueous Potassium Chloride. J. Phys. Chem 1957, 61, 1557 - 1562.

(16) Snowdon, P. N.; Turner, J. C. R.; Snowdon, P. N.; Turner, J. C. R. The concentration dependence of the Soret effect. Trans. Faraday Soc., 1960, 56, 1812-1819.

(17) Lü, H.; Leaist, D. G. Thermal-Diffusion of Tetrabutylammonium Salts in AqueousSolution at 25-degrees-C. J. Solution Chem. 1991, 20, 199 - 205.

(18) Duhr, S.; Braun, D. Why molecules move along a temperature gradient. Proc. Nat. Acad. Sci. 2006, 103, $19678-19682$.

(19) Wang, Z.; Kriegs, H.; Wiegand, S. Thermal Diffusion of Nucleotides. J. Phys. Chem. B 2012, 116, $7463-7469$.

(20) Kishikawa, Y.; Shinohara, H.; Maeda, K.; Nakamura, Y.; Wiegand, S.; Kita, R. Temperature dependence of thermal diffusion for aqueous solutions of monosaccharides , oligosaccharides , and polysaccharides. Phys. Chem. Chem. Phys. 2012, 14, 1014710153.

(21) Reichl, M.; Herzog, M.; Götz, A.; Braun, D. Why Charged Molecules Move Across a Temperature Gradient: The Role of Electric Fields. Phys. Rev. Lett. 2014, 112, 198101.

(22) Reichl, M.; Herzog, M.; Greiss, F.; Wolff, M.; Braun, D. Understanding the similarity in thermophoresis between single- and double-stranded DNA or RNA. Phys. Rev. E 2015, $\underline{91}, 062709$.

(23) Köhler, W.; Krekhov, A.; Zimmermann, W. Thermal Diffusion in Polymer Blends: Critically and Pattern Formation. Adv. Polym. Sci. 2010, 227, 145 - 198. 
(24) Stadelmaier, D.; Köhler, W. Thermal Diffusion of Dilute Polymer Solutions: The Role of Chain Flexibility and the Effective Segment Size. Macromolecules 2009, 42, 9147 9152.

(25) Putnam, S. A.; Cahill, D. G.; Wong, G. C. Temperature dependence of thermodiffusion in aqueous suspensions of charged nanoparticles. Langmuir 2007, 23, 9221-8.

(26) Sehnem, A. L.; Aquino, R.; Campos, A. F. C.; Tourinho, F. A.; Depeyrot, J.; Neto, A. M. F. Thermodiffusion in positively charged magnetic colloids: Influence of the particle diameter. Physical Review E 2014, $\underline{89}, 032308$.

(27) Colombani, J.; Bert, J.; Dupuy-Philon, J. Thermal Diffusion in (LiCl, RH2O). Journal of Chemical Physics 1999, 110, $8622-8627$.

(28) Sehnem, A. L.; Figueiredo Neto, A. M.; Aquino, R.; Campos, A. F. C.; Tourinho, F. A.; Depeyrot, J. Temperature dependence of the Soret coefficient of ionic colloids. Phys. Rev. E 2015, 92, 042311.

(29) Kita, R.; Polyakov, P.; Wiegand, S. Ludwig Soret Effect of Poly(Nisopropylacrylamide): Temperature Dependence Study in Monohydric Alcohols.

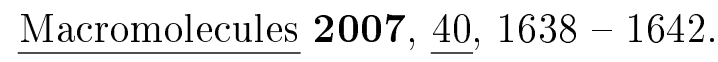

(30) Brenner, H. Is the tracer velocity of a fluid continuum equal to its mass velocity? Phys. Rev. E 2004, 70, 061201.

(31) Bielenberg, J. R.; Brenner, H. A hydrodynamic/Brownian motion model of thermal diffusion in liquids. Physica A 2005, 356, 279-293.

(32) Wiegand, S.; Ning, H.; Kriegs, H. Thermal Diffusion Forced Rayleigh Scattering Setup Optimized for Aqueous Mixtures. J. Phys. Chem. 2007, 111, 14169 - 14174.

(33) Köhler, W. Thermodiffusion in Polymer-Solutions as Observed by Forced RayleighScattering. J Chem Phys 1993, 98, 660-668. 
(34) Köhler, W.; Rosenauer, C.; Rossmanith, P. Holographic grating study of mass and thermal diffusion of polystyrene/toluene solutions. International Journal of Thermophysics 1995, 16, 11-21.

(35) Köhler, W.; Rossmanith, P. J Chem Phys 1995, 99, 5838-5847.

(36) Naumann, P.; Becker, N.; Datta, S.; Sottmann, T.; Wiegand, S. Soret Coefficient in Nonionic Microemulsions: Concentration and Structure Dependence. The Journal of

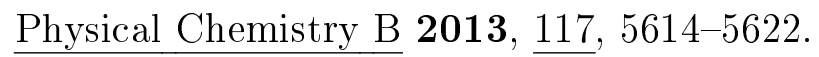

(37) Maeda, K.; Shinyashiki, N.; Yagihara, S.; Wiegand, S.; Kita, R. How does thermodiffusion of aqueous solutions depend on concentration and hydrophobicity? Eur Phys J E Soft Matter 2014, 37, 94.

(38) Kolodner, P.; Williams, H.; Moe, C. Optical measurement of the Soret coefficient of ethanol/water solutions. J. Phys. Chem. 1988, $\underline{88}, 6512-6524$.

(39) Maeda, K.; Shinyashiki, N.; Yagihara, S.; Wiegand, S.; Kita, R. Ludwig-Soret effect of aqueous solutions of ethylene glycol oligomers, crown ethers, and glycerol: Temperature, molecular weight, and hydrogen bond effect. J. Chem. Phys. 2015, 143, 124504.

(40) Niether, D.; Kawaguchi, T.; Hovancová, J.; Eguchi, K.; Dhont, J. K. G.; Kita, R.; Wiegand, S. The role of hydrogen bonding of cyclodextrin-drug complexes probed by thermodiffusion. Langmuir 2017, 33, 8483 - 8492.

(41) Bringuier, E. On the notion of thermophoretic velocity. Philosophical Magazine 2007, $\underline{87,}, 873-883$.

(42) Santos, M. P.; Gomez, S. L.; Bringuier, E.; Figueiredo Neto, A. M. Thermodiffusion in a multicomponent lyotropic mixture in the vicinity of the critical micellar concentration by using the. Phys. Rev. E 2008, 77, 011403. 
(43) Kim, J.; Tian, Y.; Wu, J. Thermodynamic and Structural Evidence for Reduced Hydrogen Bonding among Water Molecules near Small Hydrophobic Solutes. The Jornal of Physical Chemistry B 2015, 119, $12108-12116$.

(44) Mancinelli, R.; Botti, A.; Bruni, F.; Ricci, A.; Soper, A. K. Hydration of Sodium, Potassium, and Chloride Ions in Solution and the Concept of Structure Maker/Breaker. J. Phys. Chem. B 2007, 111, $13570-13577$.

(45) Eguchi, K.; Niether, D.; Wiegand, S.; Kita, R. Thermophoresis of cyclic oligosaccharides in polar solvents. Eur Phys J E Soft Matter 2016, 39, 86.

(46) Joung, I. S.; Cheatham, T. E. Determination of Alkali and Halide Monovalent Ion Parameters for Use in Explicitly Solvated Biomolecular Simulations. J. Phys. Chem. B 2008, $112,9020-9041$.

(47) Cappa, C. D.; Smith, J. D.; Wilson, K. R.; Messer, B. M.; Gilles, M. K.; Cohen, R. C.; Saykally, R. J. Effects of Alkali Metal Halide Salts on the Hydrogen Bond Network of Liquid Water. J. Phys. Chem. B 2005, 109.

(48) Huang, N.; Schlesinger, D.; Nordlund, D.; Huang, C.; Tyliszczak, T.; Weiss, T. M.; Acremann, Y.; Pettersson, L. G. M.; Nilsson, A. Microscopic probing of the size dependence in hydrophobic solvation. The Journal of Chemical Physics 2012, 136, 074507.

(49) Slusher, J. T.; Cummings, P. T. Molecular Simulation Study of Tetraalkylammonium Halides. 1. Solvation Structure and Hydrogen Bonding in Aqueous Solutions. J. Phys. Chem. B 1997, 101, $3818-3826$.

(50) Hribar-Lee, B.; Dill, K. A.; Vlachy, V. Receptacle Model of Salting-In by Tetramethylammonium Ions. The Journal of Physical Chemistry B 2010, 114, 15085-15091.

(51) Davis, J. G.; Rankin, B. M.; Gierszal, K. P.; Ben-Amotz, D. On the cooperative for- 
mation of non-hydrogen-bonded water at molecular hydrophobic interfaces. Nat Chem 2013, $\underline{5}, 796-802$.

(52) Dhont, J. K. G.; Briels, W. J. Single-particle thermal diffusion of charged colloids: Double-layer theory in a temperature gradient. Eur Phys J E Soft Matter 2008, 25, 61 $-76$.

(53) Dhont, J. K.; Wiegand, S.; Duhr, S.; Braun, D. Thermodiffusion of charged colloids: single-particle diffusion. Langmuir 2007, 23, 1674-83.

(54) Gerogiokas, G.; Calabro, G.; Henchman, R. H.; Southey, M. W. Y.; Law, R. J.; Michel, J. Prediction of Small Molecule Hydration Thermodynamics with Grid Cell Theory. Journal of Chemical Theory and Computation 2014, 10, 35 - 48.

(55) Saha, D.; Mukherjee, A. Impact of Ions on Individual Water Entropy. The Jornal of $\underline{\text { Physical Chemistry B 2016, } 120,7471-7479 .}$

(56) Saha, D.; Mukherjee, A. Connecting diffusion and entropy of bulk water at the single particle level. J, Chem. Sci. 2017, 129, 825 - 832.

(57) Agar, J. N.; Mou, C. Y.; Lin, J. L. Single-ion heat of transport in electrolyte solutions: a hydrodynamic theory. J. Phys. Chem 1989, 93, 2079-2082.

(58) Vigolo, D.; Buzzaccaro, S.; Piazza, R. Thermophoresis and thermoelectricity in surfactant solutions. Langmuir 2010, 26, 792-801.

(59) Eslahian, K. A.; Majee, A.; Maskos, M.; Wurger, A. Specific salt effects on thermophoresis of charged colloids. Soft Matter 2014, 10, 1931 - 1936.

(60) Putnam, S. A.; Cahill, D. G. Transport of Nanoscale Latex Spheres in a Temperature Gradient. Langmuir 2005, 21, $5317-5323$. 
(61) Agar, J. N. In Thermogalvanic Cells, in Advances in Electrochemistry and Electrochemical Engineering, pp. 31-121; Delahay, P., Ed.; Advances in Electrochemistry and Electrochemical Engineering, pp. 31-121; Interscience New York, 1963. 
Graphical TOC Entry 